\title{
Exploring approach motivation: Correlating self-report, frontal asymmetry, and performance in the Effort Expenditure for Rewards Task
}

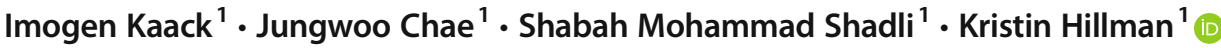

Accepted: 30 August 2020 / Published online: 15 September 2020

(C) The Psychonomic Society, Inc. 2020

\begin{abstract}
Frontal EEG asymmetry has been investigated as a physiological metric of approach motivation, with higher left frontal activity (LFA) suggested to reflect approach motivation. However, correlations between LFA and traditional metrics of approach motivation (e.g., scores from the behavioral inhibition system/behavioral approach system [BIS/BAS] survey) are inconsistent. It is also not clear how LFA correlates to approach motivation on an observable, behavioral level. Here, we tested correlations between BIS/BAS scores, LFA, and performance in the Effort Expenditure for Rewards Task (EEfRT). In our sample $(n=49)$, BIS/BAS results did not correlate to LFA values (resting or task states), and were also unrelated to EEfRT performance variables. We found evidence of significant and distinct correlations between LFA and EEfRT performance. Resting-state LFA positively correlated to effort expenditure on lower utility trials, where reward size and/or probability were suboptimal. Task-onset LFA captured in the first 5 min of the task was related to overall behavioral performance in the EEfRT. High task-onset LFA correlated to high trial completion rates, high-effort trial selection percentages, and overall monetary earnings. One interpretation of these initial findings is that resting-state LFA reflects approach tendencies to expend effort, but that this extends to suboptimal situations, whereas task-state LFA better reflects effortful approach toward high-utility goals. Given the relatively small sample size and the risk of Type I/II errors, we present the study as exploratory and the results as preliminary. However, the findings highlight interesting initial links between LFA and EEfRT performance. The need for larger replication studies is discussed.
\end{abstract}

Keywords Approach motivation $\cdot$ Frontal asymmetry $\cdot$ EEfRT $\cdot$ Effort $\cdot$ BIS/BAS

Approach motivation broadly describes incentivized behavior that stems from internal processes and/or external stimuli (Gray, 1982; Gray \& McNaughton, 2000; Harmon-Jones, Harmon-Jones, \& Price, 2013). Approach motivation is usually beneficial in that it helps an organism move toward salient outcomes or states; however, high approach motivation may contribute toward maladaptive actions, including impulsive, addictive, or aggressive behaviors (Bijttebier, Beck, Claes, \& Vandereycken, 2009; Franken, 2002; Gray, 1993; Johnson, Turner, \& Iwata, 2003; Smits \& Kuppens, 2005). The behavioral activation system (BAS) thus needs to be tempered by

Electronic supplementary material The online version of this article (https://doi.org/10.3758/s13415-020-00829-x) contains supplementary material, which is available to authorized users.

Kristin Hillman

kristin.hillman@otago.ac.nz

1 Department of Psychology, University of Otago, 275 Leith Walk, Dunedin, New Zealand the behavioral inhibition system (BIS) for optimal action, particularly when the pursuit of salient outcomes or states carries costs to the individual - for example, risk or effort (Corr, 2008; Corr \& McNaughton, 2012).

Human BIS/BAS tendencies have been widely investigated using Carver and White's (1994) self-report BIS/BAS survey. The survey provides one BIS score and three BAS subdomain scores: BAS-Drive for Reward, BAS-Fun Seeking, and BAS-Reward Responsivity. Regarding BAS subdomains, BAS-Drive for Reward items are most associated with enduring costs to achieve salient outcomes and also acting decisively (e.g., "When I want something, I usually go all-out to get it"; "If I see a chance to get something I want, I move on it right away"). BAS-Drive for Reward and related constructs can be highly adaptive if directed toward high-cost, high-reward pursuits, but these types of reward drive require adjunct self-control for optimal success (Duckworth \& Gross, 2014; Duckworth, Peterson, Matthews, \& Kelly, 2007). High BAS-Drive for Reward itself has been linked to increased externalizing behaviors (Taubitz, Pedersen, \& Larson, 2015). 
BIS/BAS scale scores are suggested to reflect stable, traitlevel tendencies, as evidenced by test- retest reliability (Cooper, Gomez, \& Aucote, 2007; Schneider, Chau, Mohamadpour, Stephens, Arya, \& Grant, 2016; Sutton \& Davidson, 1997). Trait-level approach motivation has also been linked to another metric with test-retest reliability: frontal alpha power asymmetry (Hagemann, Hewig, Seifert, Naumann, \& Bartussek, 2005; Tomarken, Davidson, Wheeler, \& Kinney, 1992; Vuga et al., 2006; Vuga, Fox, Cohn, Kovacs, \& George, 2008). In resting-state EEG recordings, greater left frontal activity (LFA) at rest — compared with right frontal activity - has been suggested to reflect a physiological index of approach motivation (Coan \& Allen, 2004; Davidson, 1984, 1998; Harmon-Jones, Gable, \& Peterson, 2010; Sutton \& Davidson, 1997). LFA correlates to selfreport BAS scores in some studies (Amodio, Master, Yee, \& Taylor, 2008; Coan \& Allen, 2003a; Harmon-Jones \& Allen 1997; Sutton \& Davidson, 1997), but not in others (Hayden et al., 2008; Hewig, Hagemann, Seifert, Naumann, \& Bartussek, 2006; Neal \& Gable, 2017; Schneider et al., 2016; Wacker, Chayanon, \& Stemmler, 2010). Detailed meta-analyses by Wacker et al. (Kuper, Käckenmester, \& Wacker, 2019; Wacker et al., 2010) have confirmed inconsistent relationships between BAS scores and LFA values. They have highlighted a wide range of effect sizes, variable "resting-state" conditions, and strong evidence for publication biases favoring significant results. With specific regard to BAS-LFA associations, these meta-analyses reveal overall mean weighted correlation values of $r=.04$ (Wacker et al., 2010) and $r=.06$ (Kuper et al., 2019) — both nonsignificant.

Discrepancies in the literature surrounding the relationship between BIS/BAS and resting-state LFA have often been attributed to methodological differences; however, self-reporting biases (Pickering, 2008; Smillie, 2008) in the BIS/BAS survey could also cloud results. Furthermore, studies demonstrating state/situational influences on the strength of LFA-approach correlations (Coan \& Allen, 2003b; Coan, Allen, \& Harmon-Jones, 2001; Crost, Pauls, \& Wacker, 2008; Rodrigues, Müller, Mühlberger, \& Hewig, 2018; Tops \& Boksem, 2010; Wacker, Mueller, Pizzagalli, Hennig, \& Stemmler, 2013) have led some to suggest that context-dependent LFA may be more indicative of approach tendencies compared with baseline resting-state LFA. In line with this capability model (Coan, Allen, \& McKnight, 2006), resting-state frontal asymmetry may provide little information about actual approach behavior. Rather, frontal activity during a goaldirected task or similar incentivized state (Pizzagalli, Sherwood, Henriques, \& Davidson, 2005; Tops \& Boksem, 2010), and/or task-related changes in frontal activity patterns during tasks (Neal \& Gable, 2019; Rodrigues et al., 2018; Rollwage, Comtesse, \& Stemmler, 2017) may be more reflective of actual behavioral tendencies.
Here, we examined whether self-reported BIS/BAS scores correlated to LFA (resting state and task state), and whether either of these measures correlated to actual performance in the Effort Expenditure for Rewards Task (EEfRT; Treadway, Buckholtz, Schwartzman, Lambert, \& Zald, 2009). In the EEfRT, participants can choose when and how to incur costs (effort and risk) to obtain virtual monetary reward. The EEfRT has been used in one LFA study (Hughes, Yates, Morton, \& Smillie, 2015), and it may represent an important untapped tool for assessing approach motivation at a behavioral level. Hughes et al. (2015) found that when resting-state LFA was recorded immediately after the EEfRT, individuals with higher LFA values had been more willing to expend effort to obtain rewards during the task - notably when the probability of payout was low. BIS/BAS was not assessed in their study, nor was there an examination of resting-state versus task-state LFA. Here, we use a combination of BIS/BAS self-report, dual state LFA, and behavioral performance in the EEfRT to test correlations between LFA and approachlike behavior. We predicted that (1) high BAS scores would correlate to higher LFA, particularly task-state LFA; (2) high BAS scores, particularly BAS-Drive for Reward scores, would correlate to higher trial completion and high-effort trial selection in the EEfRT; and (3) high LFA would correlate to higher trial completion and high-effort trial selection in the EEfRT.

\section{Method}

\section{Participants}

Seventy-five participants were initially recruited using online and print advertisements within the Department of Psychology at the University of Otago. The recruitment target was set based on a previous study examining resting-state LFA and EEfRT performance (Hughes et al., 2015), which had an initial recruitment of 55 participants and reported effect sizes of .42-.43. To detect a similar effect size in our study $(r$ $=.40,1-\beta=.80), 49$ participants would be required. Our participants received course credit for participation in the twosession experiment. A $\$ 100$ grocery store voucher was also an offer to the person who earned the most amount of EEfRT virtual money across all participants for each semester of recruitment; there were two semesters of recruitment. Of the 75 participants initially recruited, 26 were excluded from the current analysis due to missing one or more data points $(n=8)$; poor quality EEG recordings ( $n=13)$; failing inattention items $(n=2)$; or being left-handed $(n=3)$. The final data set analyzed here was comprised of 49 right-handed participants (age $20 \pm 2.7$, range: $18-31 ; 57 \%$ female). The study had received approval from the Human Ethics Committee at the University of Otago (protocol H18/004) prior to participant recruitment. 
Participants received full study information and a copy of the consent form prior to signing up for Session 1.

\section{Session 1}

In the first 1-hour session, each participant completed a written consent form, provided demographic information, and the experimenter took measures of height and weight (data not reported here). Each participant then completed a series of online questionnaires via a customized survey platform (Qualtrics ${ }^{\mathrm{XM}}$ ), which included Carver and White's (1994) BIS/BAS survey. Five other surveys were also included, but these data are not presented here: the Hospital Anxiety and Depression Scale, Perceived Stress Scale, Ten-Item Personality Inventory, International Physical Activity Questionnaire-Long Form, and a modified Self-Control Scale. Questionnaire order was randomized across participants, and five inattention items were integrated into the overall survey. Incorrect answers on two or more inattention items flagged the participant for exclusion.

\section{Session 2}

One week later, each participant arrived to the EEG lab for a 1hour session. Verbal consent was obtained, and exclusionary criteria were checked to ensure there was no history of epilepsy and that no aerobic exercise had been performed in the preceding 2 hours. Each participant was then fitted with a 32-channel wet-gel Waveguard ${ }^{\mathrm{TM}}$ cap (ANT Neurotechnology, The Netherlands) using standard 10-20 placement locations. WhiteSensor biotabs (Ambu A/S, Denmark) were applied to wrists and ankles to enable ECG capture. The EEG ground electrode (Gnd) was located between FPZ and Fz and a central reference electrode at $\mathrm{Cpz}$ for online signal referencing. All electrode impedances were targeted to $<5 \mathrm{k} \Omega$. EEG signals were sampled at $512 \mathrm{~Hz}$ and bandpass filtered online $0.5-100 \mathrm{~Hz}$. A 10-min baseline recording was performed to obtain resting-state EEG and resting-state heart rate. The heart rate measurement was performed as part of another research project; data are not reported here. Participants were instructed to relax and sit comfortably, and alternate between eyes-open and eyes-closed periods in 1-min intervals. Participants were verbally instructed when to open and close their eyes by the experimenter. Brain activity was recorded using eego ${ }^{\text {TM }}$ acquisition software (ANT Neurotechnology), and heartrate was recorded using an SE-1010 ECG machine (Edan Instruments Inc., China).

After the initial 10-min recording, the ECG electrode leads were unhooked, and ECG pads removed. The experimenter then verbally instructed the participant on how to perform the EEfRT, using visual aids and four familiarization practice trials to assist in instruction. Once the participant had completed the practice trials and indicated comfort with the parameters of task performance, the 20-min EEfRT session was initiated. The experimenter left the room during the task, but told the participant that she or she would be in the adjacent room if there were any questions or concerns, and also that the experimenter would be observing the experiment via a live video feed to ensure the correct hands/fingers were being used for the EEfRT.

The EEfRT, developed by Treadway et al. (2009), is a MATLAB (The MathWorks, Natick, MA) coded laboratory task where participants choose between high-effort (HE) and low-effort actions to earn virtual monetary reward. HE selections necessitate 100 key strokes in $21 \mathrm{~s}$ with the pinkie finger of one's nondominant hand, whereas low-effort selections necessitate 30 key strokes in $7 \mathrm{~s}$ with the index finger of one's dominant hand. For the HE task, the reward magnitude on offer varies between $\$ 1.30$ and $\$ 4.00$; for the low-effort task, the offer is a fixed value of $\$ 1.00$. Each offer also presents a probability of payout if the selected action is successfully completed-88\%, $50 \%$, or $12 \%$.

Full details of the original EEfRT, including visual examples of screen progression and generalized estimating equations for performance variables, have been described previously (Hughes et al., 2015; Treadway et al., 2009). We ran a modified version of the EEfRT, as illustrated in Fig. 1. Briefly, on each trial participants, were presented with a 1-s Fixation screen, and then an Offers screen, detailing the monetary values on offer for low-effort and high-effort actions, and the probability of payout (see Fig. 1a). Participants were then presented a Choice screen asking, "Do you choose low effort or high effort on this trial?" Participants had up to $5 \mathrm{~s}$ to assess offers and indicate their choice via a manual key stroke. Participants were then prompted to perform the selected action; a screen containing a progress bar informed real-time progress toward the required 30 or 100 key strokes associated with their respective low-effort or high-effort choice. Following Feedback and Payout screens, we presented a 2-s Reset screen to signal the start of a new trial. Trials were repetitively presented for the 20 -min session duration. Prior to the start of the EEfRT, participants were instructed that the goal of the task was to earn as much virtual money as possible in $20 \mathrm{~min}$, and they were reminded that the person who earns the most virtual money each semester would receive a real $\$ 100$ grocery store voucher at the end of the semester. At the end of the EEfRT, participants were debriefed, and their participation was complete.

\section{Data analysis}

Session 1 survey data were exported from the Qualtrics ${ }^{\mathrm{XM}}$ interface and collated within Excel (Microsoft). Session 2's EEG data were processed off-line in MATLAB R2016b using the EEGLAB toolbox (Swartz Center for Computational Neuroscience, UCSD) and custom-written scripts. For each 


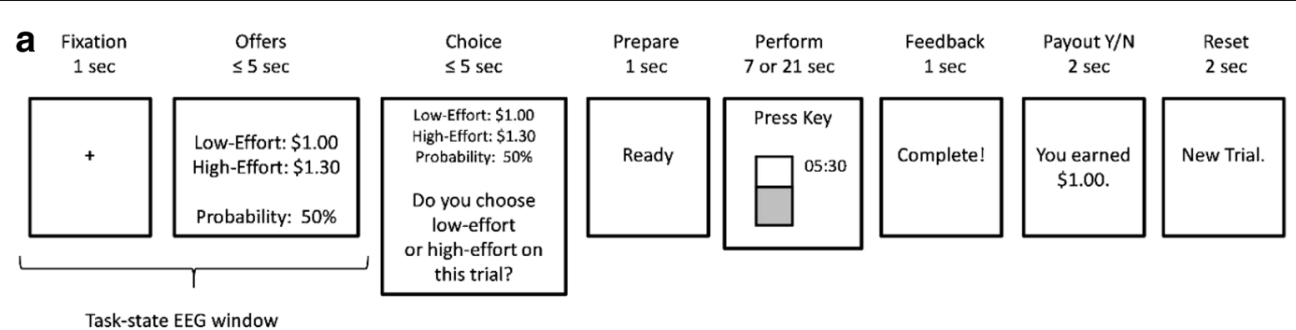

b

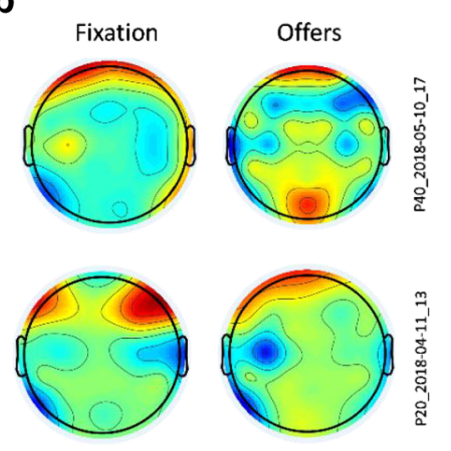

C

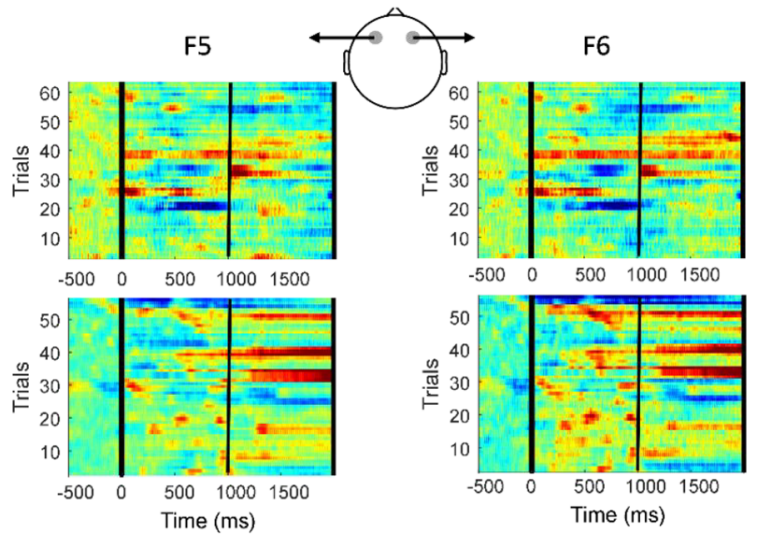

Fig. 1 Effort expenditure for rewards task (EEfRT). a We modified the original EEfRT of Treadway et al. (2009) so that each trial presented eight successive screens as illustrated above, left to right. EEG signals from the start of each trial were used to calculate task-state left frontal activity (LFA). b Scalp maps from two participants' EEfRT sessions, illustrating $10 \mathrm{~Hz}$ alpha power across the 1-s fixation phases and the first second of the offers phases. These phases were combined and treated as a single 2-s epoch for task-state LFA calculations. Hotter colors correspond to higher alpha power. $\mathbf{c}$ Time-trial spectrograms for the corresponding participants in $\mathbf{b}$, illustrating activity at the F5 and F6 electrodes during the EEfRT session. The zero time point marks the start of the trial with fixation screen appearance; the offers screen appears at 1,000 ms. Across the 49 participants, there were no consistent patterns of activation seen when comparing fixation phases to offers phases. (Color figure online) participant, recordings were first loaded into MATLAB and bandpass filtered 2-60 Hz. All 10 min of the resting-state EEG was used to comprise the resting-state signal. The task-state signal from the 20-min EEfRT was first split into four segments of interest: task-onset (0-5 min), mid-task (8-13 min), end-of-task (15-20 min), and full-task signal (0-20 min). In our original experimental design it was the signal from the first 5 min of the EEfRT ("task onset") that we were particularly interested in, as this should capture entrance into a task state, as compared with the preceding 10-min resting-state period.

Within each segment of interest, each trial was identified and the first $2 \mathrm{~s}$ of signal (1-s Fixation screen +1 -s Offers screen; see Fig. 1) was extracted and concatenated to form the task signal for that segment. This was made possible by an event stamp that marked the start of the Fixation screen, setting the zero time point for the EEG window. This window was selected as there is task engagement (fixation, assessment of offers), but no motoric key-stroke activity. The $500 \mathrm{~ms}$ preceding the Fixation event stamp was used for baseline subtraction. It is appreciated that the psychological processes underlying fixation will differ from those underlying assessment of offers, however, the two were purposefully merged to create a composite "task-state" signal. In all likelihood the "resting-state" signal is not composed of a single, uniform psychological state, but rather represents a composite of diverse psychological processes (e.g., visual fixation around the room, daydreaming), so using a combined window for the "task state" provided a composite-composite comparison between states. As illustrated in Fig. 1b-c, alpha power and frontal activity varied from the Fixation phase to the Offers phase, but across all 49 participants there were no consistent patterns.

Prepared signals were loaded and channels rereferenced off-line against the M1 and M2 mastoid electrodes. EEG signals were split into 1-s, nonoverlapping epochs for data rejection. Independent component analysis with ADJUST 1.1 (Mognon, Jovicich, Bruzzone, \& Buiatti, 2011) was used to remove common artifacts, and epochs were then visually inspected; the signal was manually cut by the experimenter if channels of interest (F3-F6) contained high-amplitude muscle or movement artifact, obvious blink artifacts, or sudden spurious changes in the signal. The resultant data were split into 2-s Hanning window epochs with $50 \%$ overlap. There were $504 \pm 83$ epochs available for the resting state, taken from an equal combination of eyes open and eyes closed; 57 \pm 38 epochs for task onset; $64 \pm 31$ epochs for mid-task; $64 \pm$ 41 epochs for end-of-task; and $215 \pm 64$ epochs for full task.

A Fourier transform was applied and spectral power in the alpha band $(8-13 \mathrm{~Hz})$ calculated for each frontal channel. 
Frequency resolution for the final spectrum was $0.5 \mathrm{~Hz}$. Six frequencies were extracted from the specified $8-13 \mathrm{~Hz}$ range. The epoch time points used to quantify alpha power were 0 to $2 \mathrm{~s}$. Alpha power values were natural log transformed, and a composite LFA score was computed from $([\mathrm{F} 6-\mathrm{F} 5]+[\mathrm{F} 4-$ F3]) / 2. A composite of frontal electrodes was selected to replicate the methodology of Hughes et al. (2015). Alpha power in the frontal cortex signal is traditionally interpreted as the inverse of active cortical processing (Allen, Coan, \& Nazarian, 2004; Laufs et al., 2003) - that is, higher alpha power in right hemisphere electrodes F6 and F4 would be interpreted as lower levels of cortical processing in right frontal areas. Thus, in our LFA calculation, a value $>0$ corresponds to higher alpha power in right hemisphere sites (F6, F4) as compared with left hemisphere sites (F5, F3), meaning that left frontal sites are capturing more active cortical processing.

Custom-written MATLAB scripts were used to sort the EEfRT behavioral data along the lines of effort (high, low); reward magnitude (high $>\$ 3$, medium $\$ 2.01-\$ 3.00$, and low $<\$ 2$ ); and probability of reward (high $88 \%$, medium $50 \%$, low $12 \%)$. All data were statistically analyzed in GraphPad (Prism) and JASP (Version 0.11.1). Normality was assessed using the Shapiro-Wilk test, Bayesian Pearson or Kendall's tau correlations were performed accordingly, and these results are presented in the main manuscript. Since a number of previous studies in the literature have predominately reported Pearson's $r$ with associated $p$ values, we provide Pearson correlation reports for all data (see the Supplemental Information) should ad hoc comparisons between studies be desired. Bayes factors $\mathrm{B}_{10}>10$ were considered as significant correlations. Planned comparisons between two variables were via $t$ test, and comparisons between three or more variables were assessed using an analysis of variance (ANOVA), with repeated measures where appropriate. Post hoc multiple comparisons were made using Tukey's test. For all comparisons, significance was assumed at $p<.05$.

\section{Results}

\section{Self-report survey measures of approach motivation}

The BIS/BAS survey was scored according to Carver and White (1994), with three subdomains assigned to BAS: BAS-Drive for Reward, BAS-Fun Seeking, and BASReward Responsivity. Within our sample, BAS subdomains exhibited positive correlations with one another and acceptable internal consistencies (see Table 1, $n=49$; Cronbach's $\alpha$ s $\geq$.68). BIS scores were negatively correlated to all BAS subdomains, with a significant relationship observed between BIS and BAS-Fun Seeking. As mentioned in the Method section, Kendall's correlations were used when data sets did not meet the assumption of normality. For these data sets, we additionally provide Pearson's $r$ values (see Supplemental Information) should ad hoc comparisons with previous studies be desired.

\section{Objective measures of LFA and effortful performance}

Resting-state LFA has been posited as an index of trait-level approach motivation, with higher LFA correlating to higher approach motivation (Coan \& Allen, 2003a; Harmon-Jones \& Allen, 1997; Sutton \& Davidson, 1997). In our participant pool, we assessed resting-state LFA and then task-state LFA during specified segments of the 20-min EEfRT (see Table 2). While resting-state LFA positively correlated to certain aspects of task-state LFA, mean LFA values did differ between analysis segments, $F(2.9,140.7)=18.4, p<.001$ (see Fig. 2). Post hoc Tukey's multiple comparisons revealed that taskonset LFA was significantly higher than all other segments $(p \leq .03)$, and end-of-task LFA was significantly lower than all other segments $(p \leq .005)$.

EEfRT performance variables of interest are shown in Table 3. Participants who successfully completed a higher percentage of their selected trials earned more virtual money in the 20-min session. Longer decision times (i.e., deciding whether to go low-effort or HE on presentation of the Choice screen; see Fig. 1) were associated with fewer trial initiations overall in the 20-min session, but higher \% selection of HE offers. Percentage of trials completed was used as a variable to gauge completion of goal-directed behavior, since BAS-Drive for Reward assays one's motivation to follow through with set goals. Likewise, decision time was examined as a variable given BAS-Drive for Reward queries impetus to act ("If I see a chance to get something I want, I move on it right away"; Carver \& White, 1994).

To gain a better understanding of the types of HE trials participants were selecting, and how this related to overall earnings in the task, we broke down the HE trials into subcategories based on reward size payout (high $>\$ 3$, medium $\$ 2.01-\$ 3.00$, or low $<\$ 2$ ) and probability of payout (high $88 \%$, medium $50 \%$, or low $12 \%$ ). The majority of participants' HE choices occurred when the HE option was associated with a high reward payout, $F(2,144)=282, p<.001$, or high-probability of payout, $F(2,144)=173, p<.001$ (see Fig. $3 a)$. Total money earned in the task increased alongside selection of HE, high-probability offers, $r(47)=.50, p<.001$ (see Fig. 3b). Selection of HE, low-probability offers was inversely related to total monetary earnings, $r(47)=-.55, p<.001$. Trends in the same directions were seen in relation to reward size payout: total money earned versus $\%$ selection of HE, high-reward offers, $r(47)=.26, p=.08$, and total money earned versus \% selection of HE, low-reward offers, $r(47)=$ $-.29, p=.04$. There was no relationship between total money earned and \% selection of HE, medium-probability trials, 
Table 1 Kendall's correlations between BIS/BAS domain scores

\begin{tabular}{|c|c|c|c|c|c|}
\hline $\begin{array}{l}\text { Mean } \pm S D \\
{[\text { Range] }}\end{array}$ & & $\begin{array}{l}\text { BAS Drive } \\
11 \pm 2.3 \\
{[5-16]}\end{array}$ & $\begin{array}{l}\text { BAS Fun Seeking } \\
12 \pm 2.3 \\
{[8-19]}\end{array}$ & $\begin{array}{l}\text { BAS Reward } \\
18 \pm 2.1 \\
{[11-20]}\end{array}$ & $\begin{array}{l}\text { BIS } \\
22 \pm 3.1 \\
{[15-28]}\end{array}$ \\
\hline \multirow[t]{2}{*}{ BAS Drive } & $\tau$ & - & .15 & $.36 * * *$ & -.04 \\
\hline & $\mathrm{BF}_{10}$ & - & .58 & 141 & .20 \\
\hline \multirow[t]{2}{*}{ BAS Fun } & $\tau$ & & - & .26 & $-.32 * *$ \\
\hline & $\mathrm{BF}_{10}$ & & - & 4.9 & 35 \\
\hline \multirow[t]{2}{*}{ BAS Reward } & $\tau$ & & & - & -.12 \\
\hline & $\mathrm{BF}_{10}$ & & & - & .37 \\
\hline
\end{tabular}

$* \mathrm{BF}_{10}>10, * * \mathrm{BF}_{10}>30, * * * \mathrm{BF}_{10}>100$

$r(47)=-.06, p=.69$, or HE, medium-reward trials, $r(47)=$ $-.06, p=.64$.

Examination of the highest and lowest quartiles of money earned confirmed that participants who earned the most virtual money ( $n=12, \$ 66.53 \pm \$ 2.01)$ had selected more HE options, notably on high-reward, high-probability offers, as compared with those who had earned the least virtual money $(n=$ $12, \$ 35.77 \pm \$ 5.8$; see Fig. $3 \mathrm{c}$ ). The high earners also successfully completed more of their selected actions (whether HE or low effort) as compared with the low earners, and high earners in our cohort never selected HE on low-reward, lowprobability offers.

\section{Correlations between BIS/BAS, LFA and effortful performance}

Our first a priori prediction was that high BAS scores would correlate to higher LFA, particularly task-state LFA. In our sample, BIS/BAS component scores showed no significant relationship to resting-state LFA, nor to any of the task-state LFA measures (see Table 4). Our second prediction was that high BAS scores, particularly BAS-Drive for Reward scores, would correlate to higher trial completion rates and higher $\%$
HE trial selection in the EEfRT. As shown in Table 4, BIS/ BAS component scores were not statistically related to any of the performance variables in the EEfRT. Our third prediction was that higher LFA would correlate to higher trial completion rates and higher \% HE trial selection in the EEfRT. As shown in Table 5, resting-state LFA did not correlate to performance variables in the 20-min EEfRT. There were, however, significant correlations between task-state LFA and EEfRT performance.

Task-onset LFA (0-5 $\mathrm{min}$ ) correlated to the total money earned in the EEfRT, as well as percentage of trials completed and \% HE selections across the 20-min session. Task-onset LFA did not correlate to performance variables within the first 5 min of the EEfRT (see Table 5, right). Full-task LFA (0-20 min) also correlated to the $\%$ of HE selections across the 20min session; participants who exhibited a higher $\%$ of $\mathrm{HE}$ choice selections also exhibited a higher full-task LFA value.

To examine if BIS/BAS scores and/or LFA measures were related to the more nuanced aspects of the EEfRT (i.e., influence of reward size or probability on $\mathrm{HE}$ action selection), we used the high-medium-low subcategories described above. BAS-Fun Seeking was significantly associated with HE choice selection on medium probability offers, $r(47)=.41, p=.02$. No

Table 2 Pearson correlations between LFA segments

\begin{tabular}{|c|c|c|c|c|c|c|}
\hline $\begin{array}{l}\text { Mean } \pm S D \\
\text { [Range] }\end{array}$ & & $\begin{array}{l}\text { Resting } \\
.06 \pm .11 \\
{[-.15-.41]}\end{array}$ & $\begin{array}{l}\text { Task onset } \\
.13 \pm .15 \\
{[-.12-.41]}\end{array}$ & $\begin{array}{l}\text { Mid-task } \\
.08 \pm .11 \\
{[-.21-.35]}\end{array}$ & $\begin{array}{l}\text { End-of-task } \\
-.01 \pm .12 \\
{[-.22-.37]}\end{array}$ & $\begin{array}{l}\text { Full task } \\
.06 \pm .10 \\
{[-.13-.33]}\end{array}$ \\
\hline \multirow[t]{2}{*}{ Resting } & $r$ & - & $.50 * * *$ & $.52 * * *$ & .32 & $.74 * * *$ \\
\hline & $\mathrm{BF}_{10}$ & - & 123 & 195 & 1.9 & $>1,000$ \\
\hline \multirow[t]{2}{*}{ Task onset } & $r$ & & - & $.66 * * *$ & .36 & $.78 * * *$ \\
\hline & $\mathrm{BF}_{10}$ & & - & $>1,000$ & 3.7 & $>1,000$ \\
\hline \multirow[t]{2}{*}{ Mid-task } & $r$ & & & - & .23 & $.63 * * *$ \\
\hline & $\mathrm{BF}_{10}$ & & & - & .59 & $>1,000$ \\
\hline \multirow[t]{2}{*}{ End-of-task } & $r$ & & & & - & $0.56 * * *$ \\
\hline & $\mathrm{BF}_{10}$ & & & & - & 979 \\
\hline
\end{tabular}

$* \mathrm{BF}_{10}>10, * * \mathrm{BF}_{10}>30, * * * \mathrm{BF}_{10}>100$ 


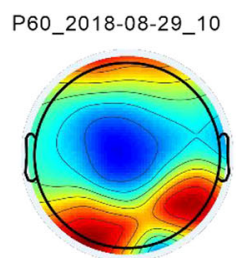

resting

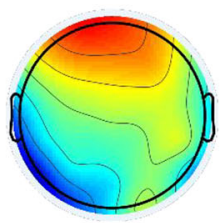

task-onset

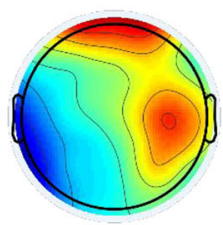

mid-task

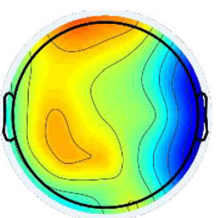

end-of-task

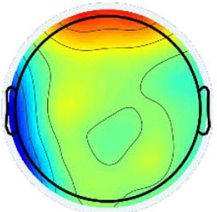

full task

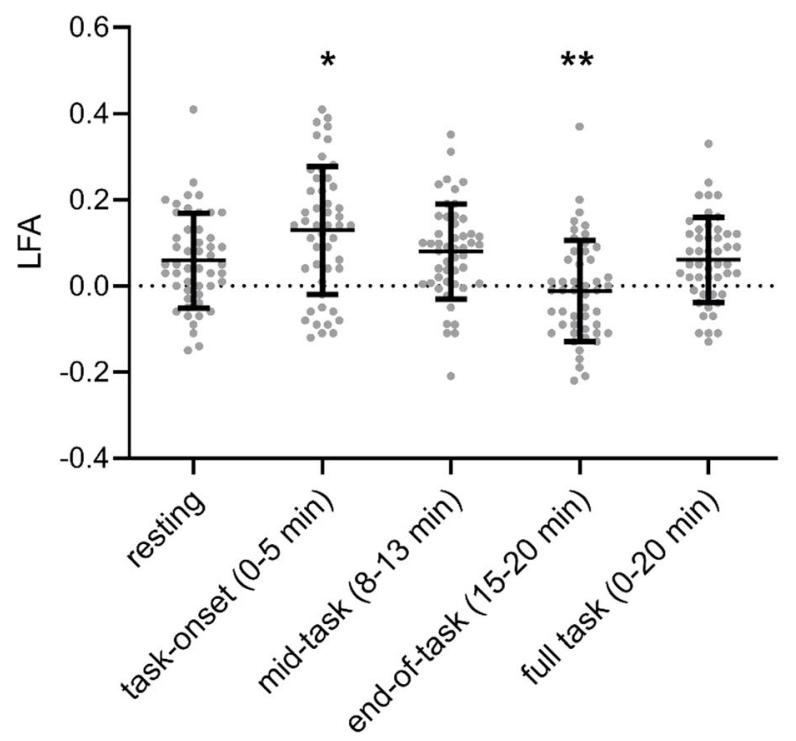

Fig. 2 Left frontal activity (LFA) comparisons between segments. Scalp maps from one representative participant are shown to illustrate changes in $10-\mathrm{Hz}$ alpha power across EEfRT recording segments. Hotter colors correspond to higher alpha power. Data from all participants are shown in the plot. Mean LFA increased from resting state to task onset. Task-onset

other significant relationships between BIS/BAS scores and EEfRT HE choice behavior were found. Resting-state LFA significantly correlated to $\mathrm{HE}$ choice behavior on medium probability offers, $r(47)=.38, p=.009$, as well as medium reward offers, $r(47)=.41, p=.008$ (see Fig. 4a). Resting-
LFA was also significantly higher than all other task-related segments. End-of-task LFA was significantly lower than all other segments of interest. Data are shown as mean $\pm S D$, $* \mathrm{p}<.05$, ** $<<.01$. (Color figure online)

state LFA also significantly correlated to HE choice behavior on low-probability offers, $r(47)=.37, p=.02$ (see Fig. 4b). There was no resting-state LFA difference between the highest earning quartile (LFA $0.032 \pm 0.09$ ) and the lowest earning quartile $(0.057 \pm 0.09)$ in the EEfRT ( $p=.29$; see Fig. $4 c)$.

Table 3 Kendall's correlations between EEfRT performance variables

\begin{tabular}{|c|c|c|c|c|c|c|}
\hline $\begin{array}{l}\text { Mean } \pm S D \\
\text { [Range] }\end{array}$ & & $\begin{array}{l}\text { Total \$ } \\
56 \pm 13 \\
{[25-71]}\end{array}$ & $\begin{array}{l}\text { Trials } \\
63 \pm 7.6 \\
{[44-78]}\end{array}$ & $\begin{array}{l}\% \text { completed } \\
96 \pm 6.5 \\
{[74-100]}\end{array}$ & $\begin{array}{l}\% \mathrm{HE} \\
38 \pm 18 \\
{[0-100]}\end{array}$ & $\begin{array}{l}\text { Decision (sec) } \\
1.4 \pm 0.34 \\
{[.38-2.4]}\end{array}$ \\
\hline \multirow[t]{2}{*}{ Total \$ } & $\tau$ & - & -.11 & $.53 * * *$ & .23 & -.07 \\
\hline & $\mathrm{BF}_{10}$ & - & .35 & $>1000$ & 2.6 & .23 \\
\hline \multirow[t]{2}{*}{ Trials } & $\tau$ & & - & -.12 & $-.84 * * *$ & $-.42 * * *$ \\
\hline & $\mathrm{BF}_{10}$ & & - & .37 & $>1000$ & $>1000$ \\
\hline \multirow[t]{2}{*}{$\%$ completed } & $\tau$ & & & - & .27 & -.03 \\
\hline & $\mathrm{BF}_{10}$ & & & - & 2.4 & .19 \\
\hline \multirow[t]{2}{*}{$\% \mathrm{HE}$} & $\tau$ & & & & - & $.28 *$ \\
\hline & $\mathrm{BF}_{10}$ & & & & - & 10 \\
\hline
\end{tabular}

$\mathrm{HE}=$ high effort, $* \mathrm{BF}_{10}>10, * * \mathrm{BF}_{10}>30, * * * \mathrm{BF}_{10}>100$ 


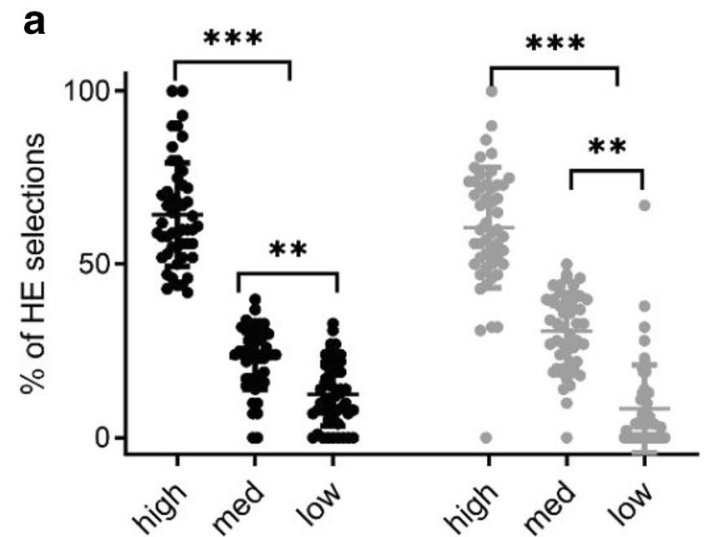

Reward \$

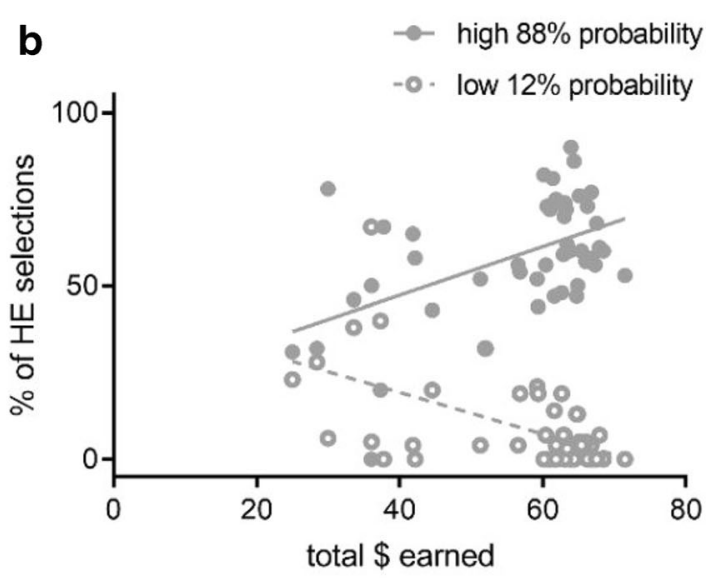

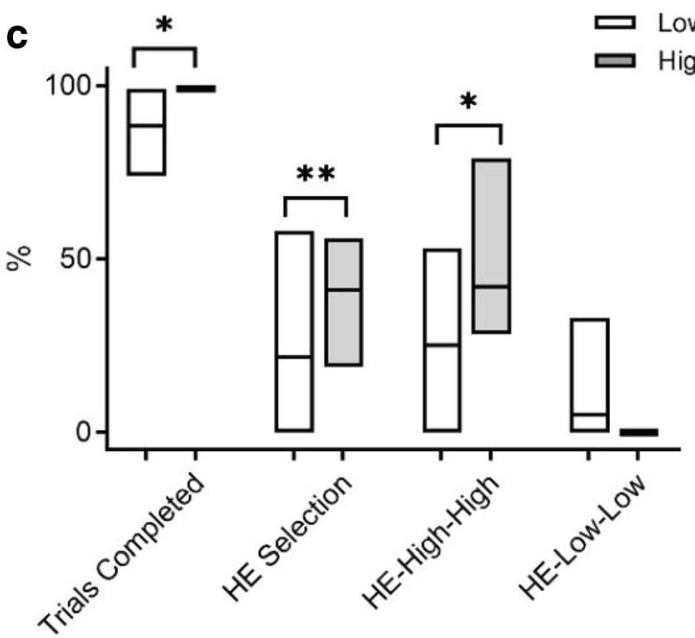

Fig 3 Characterization of high-effort (HE) choices. a The majority of HE selections occurred on trials where a high reward $(>\$ 3)$ or high probability of payout $(88 \%)$ was presented. b The overall amount of money earned in the EEfRT session was significantly associated with HE selection on high-probability offers and HE avoidance on low-probability offers. There was no correlation between medium-probability $(50 \%)$
HE selection and money earned; data not shown. c High earners in our cohort selected HE trials more often, particularly on HE, high-reward, high-probability offers (HE-High-High). The high earner quartile examined here $(n=12)$ never choose HE, low-reward, low-probability offers (HE-Low-Low). Boxes span min to max, with a line indicating the mean

Table 4 Kendall's correlations between BIS/BAS, LFA, and EEfRT performance

\begin{tabular}{|c|c|c|c|c|c|c|c|c|c|c|}
\hline & & \multicolumn{5}{|l|}{ LFA } & \multicolumn{4}{|l|}{ EEfRT } \\
\hline & & Resting & Task onset & $\begin{array}{l}\text { Mid- } \\
\text { task }\end{array}$ & $\begin{array}{l}\text { End-of- } \\
\text { task }\end{array}$ & Full task & Total \$ & $\%$ complete & $\% \mathrm{HE}$ & Decision (sec) \\
\hline \multirow[t]{2}{*}{ BAS Drive } & $\tau$ & .03 & .09 & -.002 & -.03 & .05 & -.01 & .14 & .001 & -.22 \\
\hline & $\mathrm{BF}_{10}$ & .19 & .28 & .19 & .19 & .21 & .19 & .52 & .19 & 2.3 \\
\hline \multirow[t]{2}{*}{ BAS Fun } & $\tau$ & .03 & -.12 & -.12 & -.11 & -.17 & -.06 & .08 & .15 & .09 \\
\hline & $\mathrm{BF}_{10}$ & .19 & .37 & .40 & .36 & .76 & .23 & .26 & .58 & .28 \\
\hline \multirow[t]{2}{*}{ BAS Reward } & $\tau$ & .17 & .09 & -.02 & .12 & .007 & .01 & .21 & -.03 & -.07 \\
\hline & $\mathrm{BF}_{10}$ & .83 & .29 & .19 & .39 & .19 & .19 & 1.5 & .19 & .23 \\
\hline \multirow[t]{2}{*}{ BIS } & $\tau$ & .03 & -.05 & .07 & .03 & .06 & .05 & .02 & -.09 & .05 \\
\hline & $\mathrm{BF}_{10}$ & .19 & .21 & .24 & .19 & .22 & .21 & .19 & .29 & .21 \\
\hline
\end{tabular}

LFA = left frontal activity; EEfRT = Effort Expenditure for Rewards Task; no significant results were observed 
Table 5 Kendall's correlations between LFA segments and EEfRT performance

\begin{tabular}{|c|c|c|c|c|c|c|c|c|c|}
\hline & & \multicolumn{4}{|c|}{ Overall performance across $20 \mathrm{~m}$} & \multicolumn{4}{|c|}{ Performance during 5-min segment } \\
\hline & & Total \$ & $\%$ complete & $\% \mathrm{HE}$ & Decision (sec) & Total \$ & $\%$ complete & $\% \mathrm{HE}$ & Decision $(\mathrm{sec})$ \\
\hline \multirow[t]{2}{*}{ Resting } & $\tau$ & -.10 & -.14 & .24 & .19 & & & & \\
\hline & $\mathrm{BF}_{10}$ & .22 & .27 & .68 & .39 & & & & \\
\hline \multirow[t]{2}{*}{ Task onset } & $\tau$ & $.33 *$ & $.37 *$ & $.43 *$ & .11 & .11 & .22 & .02 & .21 \\
\hline & $\mathrm{BF}_{10}$ & 16 & 17 & 29 & .23 & .18 & 1.4 & .21 & 3.4 \\
\hline \multirow[t]{2}{*}{ Mid-task } & $\tau$ & .16 & .24 & .28 & .21 & .03 & .14 & .09 & .12 \\
\hline & $\mathrm{BF}_{10}$ & .32 & .67 & 1.1 & .46 & .16 & .54 & .39 & .35 \\
\hline \multirow[t]{2}{*}{ End-of-task } & $\tau$ & .22 & .03 & .02 & .08 & .16 & .21 & .04 & -.05 \\
\hline & $\mathrm{BF}_{10}$ & .57 & .18 & .18 & .21 & .38 & .61 & .27 & 1.9 \\
\hline \multirow[t]{2}{*}{ Full task } & $\tau$ & .22 & .15 & $.38 *$ & .11 & & & & \\
\hline & $\mathrm{BF}_{10}$ & .54 & .30 & 18 & .23 & & & & \\
\hline
\end{tabular}

$* \mathrm{BF}_{10}>10 ; * * \mathrm{BF}_{10}>30 ; * * * \mathrm{BF}_{10}>100$

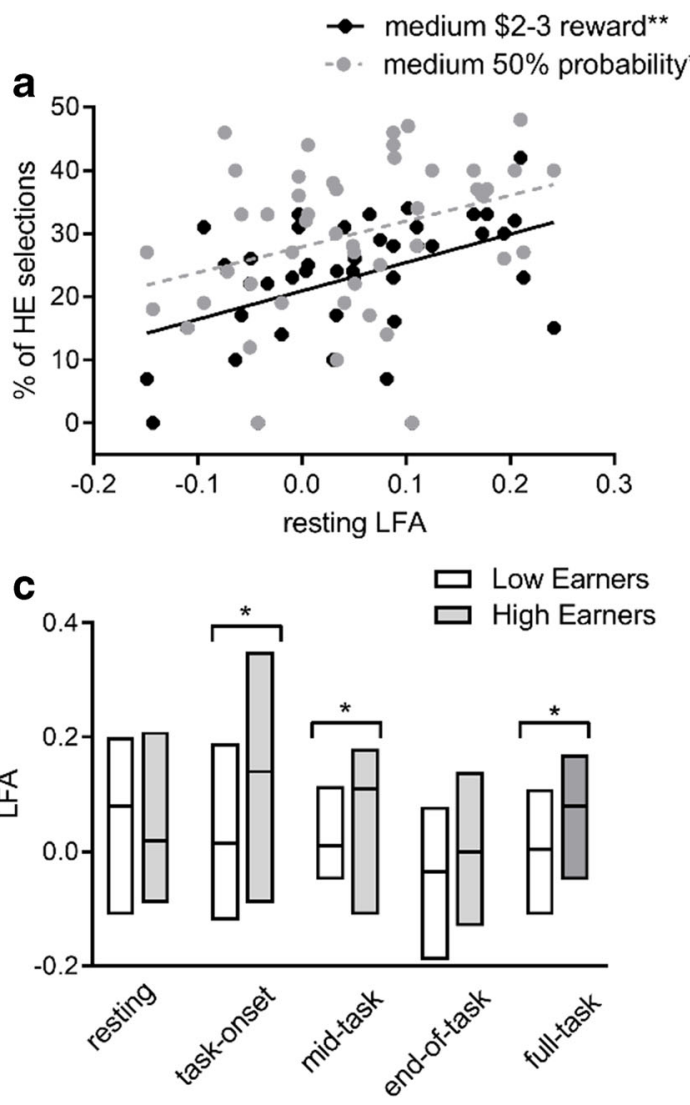

Fig. 4 Left frontal activity (LFA) and Effort Expenditure for Rewards Task (EEfRT) performance. a Resting-state LFA positively correlated to high-effort (HE) choices on medium reward and medium probability offers. b Resting-state LFA also correlated to HE choices on lowprobability offers. c Task-onset, mid-task, and full-task LFA values were significantly different between highest and lowest earning quartiles in the EEfRT. Boxes span min to max, with a line indicating the mean. d Scalp maps from two representative participants - one from the low earning quartile and one from the high earning quartile-illustrating the shift in

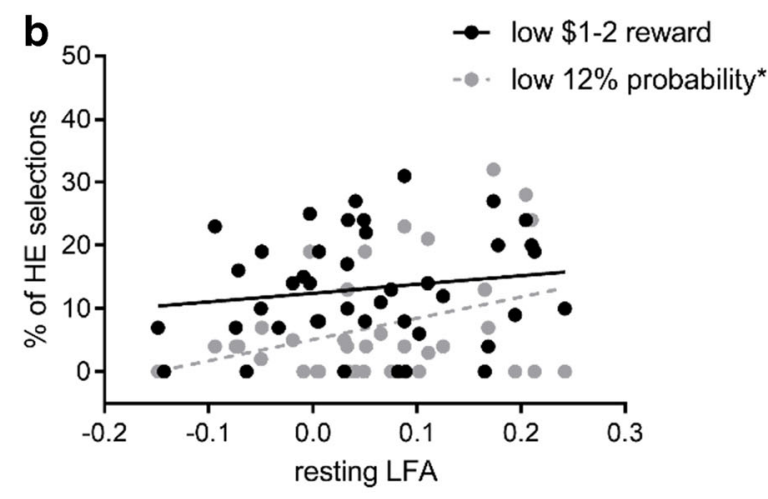

d
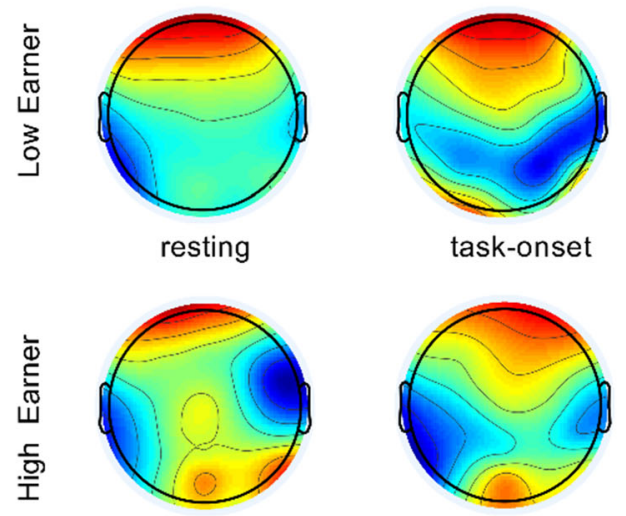

frontal alpha seen at task onset in high earners. Heat maps illustrate alpha power at $10 \mathrm{~Hz}$, with hotter colors corresponding to higher alpha power. Other participants from the high earning quartile had more pronounced shifts in alpha power during task onset; the two participants shown in $\mathbf{d}$ were selected for comparison because they had a similar trial count, and $\%$ HE trial selection, within the 5-min task-onset segment (participant in top row performed 13 trials, five were HE; participant in bottom row performed 12 trials, five were HE). (Color figure online) 
Task-state LFA values showed no significant associations with HE selection on medium-reward or low-reward offers (all $p \mathrm{~s} \geq .11$ ), nor with HE selection on medium-probability or low-probability offers (all $p \mathrm{~s} \geq .58$ ). However, there were significant differences in LFA between the lowest earning quartile and the highest earning quartile at task onset $(0.02 \pm$ 0.11 vs. $0.13 \pm 0.13, p=.048)$, mid-task $(0.02 \pm 0.05$ vs. 0.09 $\pm 0.08, p=.04)$, and across the full task $(0.008 \pm 0.06$ vs. 0.08 $\pm 0.06, p=.01)$.

To assess the robustness of our findings, we ran post hoc power calculations, focusing on the LFA-EEfRT correlations of interest. As stated in the Method section, an a priori power calculation had informed our sample size of 49 . Based on this sample size and our observed correlation coefficients, two of our significant findings exceeded $80 \%$ power (see Table 6). Of the remaining analyses, post hoc power values ranged between $64 \%$ and $77 \%$. Post hoc power calculations can be divisive, particularly when based on observed effect sizes (e.g., see Gelman, 2019; Lenth, 2001). However, we provide such calculations here in attempts to gauge how many participants would be needed to achieve $\beta=0.2$, as compared with our cohort of $n=49$.

\section{Discussion}

Overall, we did not find a significant correlation between LFA and approach motivation as assessed by the BIS/BAS survey. We captured resting-state LFA and task-state LFA in our study, given the increasing interest in trait versus state differences in frontal asymmetry (Coan \& Allen, 2003b; Coan et al., 2006); however, none of the LFA measures correlated to BIS/ BAS component scores. We did find significant and distinct relationships between LFA and EEfRT performance in our sample: (1) resting-state LFA recorded pretask correlated to effort expenditure on lower utility offers (i.e., those that had only mid-range reward values, and/or those that had less certainty of payout), whereas (2) task-onset LFA recorded in the first $5 \mathrm{~min}$ of the EEfRT correlated to astute effort-outcome decision-making and optimal monetary earnings overall.

In isolation, our results from the BIS/BAS survey are consistent with previous reports. Our sample's BIS/BAS mean scores and ranges are in line with those of other university student samples (e.g., Coan \& Allen, 2003a; Sutton \& Davidson, 1997), and our sample's BAS subdomain scores were significantly correlated with one another, in line with Carver and White (1994). We did not pool the BAS subdomains into a single BAS score because of our specific interest in BAS-Drive. Similar to the BIS/BAS metrics, our sample's LFA values were also consistent with previous reports examining LFA in university students (e.g., Hughes et al., 2015; Neal \& Gable, 2017). Hence, we are confident that the BIS/BAS survey and LFA assessment worked as intended; however, we found no significant relationship between the two. This adds another null finding to the existing mix of null and positive correlations reported in the literature when attempting to link self-report measures (BIS/BAS) with physiological LFA measures (see Kuper et al., 2019; Wacker et al., 2010).

Despite its long-history of use, the BIS/BAS survey may not be the best metric for capturing approach motivation (Cogswell, Alloy, van Dulmen, \& Fresco, 2006; Corr, 2013; Corr, 2016; Smillie, Pickering, \& Jackson, 2006; Torrubia, Avila, Molto, \& Caseras, 2001). There appears to be a shift in tactics in recent approach motivation studies in this regard. For example, Rollwage et al. (2017) used the BIS/BAS in combination with a battery of other psychometric scales and iterated principal factor analysis to generate BAS factor scores. The Reinforcement Sensitivity Theory of Personality Questionnaire (RST-PQ; Corr, 2016; Corr \& Cooper, 2016) is also gaining traction, stemming from continued evolution of the reinforcement sensitivity theory (Corr, 2008; Corr \& McNaughton, 2012; Gray \& McNaughton, 2000). The RSTPQ has been employed in one recent LFA-related study (De Pascalis, Sommer, \& Scacchia, 2018), and in hindsight this may have been a useful survey to use in our study, either in isolation or as an adjunct to the BIS/BAS.

Table 6 Post hoc power calculation for observed correlation coefficients

\begin{tabular}{llll}
\hline & $\begin{array}{l}\text { Observed } \\
\tau / r\end{array}$ & $N$ needed for $\beta=0.2$ & Power at $n=49$ \\
\hline Task-onset LFA and \% HE & 0.43 & 40 & $88 \%$ \\
Task-onset LFA and \% complete & 0.37 & 55 & $75 \%$ \\
Task-onset LFA and total \$ earned & 0.33 & 70 & $64 \%$ \\
Full-task LFA and \% HE & 0.38 & 52 & $77 \%$ \\
Resting-state LFA and HE, medium-probability trials & 0.38 & 52 & $77 \%$ \\
Resting-state LFA and HE medium-reward trials & 0.41 & 44 & $84 \%$ \\
Resting-state LFA and low-probability trials & 0.37 & 55 & $75 \%$ \\
\hline
\end{tabular}

LFA = left frontal activity; $\mathrm{HE}=$ high effort 
Irrespective of the survey(s) used, surveys are nevertheless reliant on introspection and vulnerable to self-reporting biases (Pickering, 2008; Smillie, 2008). For this reason, we wanted to incorporate a performance-based metric of approach motivation in our study. We used a modified version of the EEfRT designed by Treadway et al. (2009), given the task taps into multiple facets of the BAS including incentivized action, incurring action costs, and goal-drive persistence (Corr, 2013; Corr \& Cooper, 2016; Gray \& McNaughton, 2000; HarmonJones et al., 2013). If LFA reflects behavioral approach motivation, we predicted that individuals with high LFA would work diligently in the EEfRT (i.e., high trial completion) and expend more effort in the task (\% HE selection).

We found no correlation between resting-state LFA and trial completion, or between resting-state LFA and HE choice percentage. However, we did find significant correlations between task-state LFA (specifically, task-onset LFA and fulltask LFA) and HE choice percentage. Higher LFA values recorded in the initial $5 \mathrm{~min}$ of the task were associated with higher HE choice percentages over the entire 20-min session, higher completion of selected actions, and higher total monetary earnings overall. However, the statistical power associated with the latter two correlations ( $75 \%$ and $64 \%$, respectively) fell below the generally accepted criterion of $80 \%$; this limitation is discussed below.

Full-task LFA - calculated across the 20-min sessionwas also associated with higher HE choice percentage in our cohort $(\beta=0.23)$. If we had observed a correlation only between full-task LFA and overall HE choice percentage, then it could be argued that frontal asymmetry is reflecting some motoric aspect of HE responding or perhaps is a direct consequence of HE responding. However, we observed an increase in LFA in the first 5 min of the task, as compared with the preceding resting-state LFA, and this was not statistically related to the HE responding going on in those $5 \mathrm{~min}$. Rather, this task-onset LFA correlated to overall effective performance in the EEfRT. It is this task-onset LFA that we highlight as an interesting finding, as this may represent a trait (resting state) to state (task onset) shift in LFA that may be useful in future studies of approach motivation.

While our resting-state LFA measures were not correlated to overall HE choice percentage, resting-state LFA was related to HE choice selection on HE offers that presented a medium reward $(\$ 2.01-\$ 3.00$, versus a low $<\$ 2$, or high $>\$ 3$, reward). BAS-Fun Seeking scores also correlated to HE choice selection on these types of mid-range offers. Resting-state LFA in our cohort was also related to HE choice selection on HE offers that presented medium or low probabilities of payout ( $50 \%$ or $12 \%$ chance of payout, respectively). Though, again, the observed power of these correlations fell below criterion ( $\beta=0.23$ and 0.25 , respectively). Of note, these types of $\mathrm{HE}$ choice selection were not associated with optimal monetary earnings. Indeed, HE choice selection on low-probability trials was inversely correlated to overall earnings. BAS-Drive for Reward scores did not correlate to any EEfRT metric, which was not in line with our original prediction.

One interpretation of these results is that resting-state LFA is reflective of a specific facet of trait-level approach motivation-a willingness/eagerness to expend effort. However, as illustrated here by the EEfRT, such impetus is not necessarily functionally advantageous in all scenarios. The observed correlation between HE medium-reward choice selection and BAS-Fun Seeking scores suggests that, at least in part, this eagerness to expend effort may stem from enjoyment/novelty of action; however, since the BAS-Fun Seeking score correlation did not extend to the HE medium/ low-probability choices, this becomes difficult to interpret in our specific study. Use of the RST-PQ may have provided some clarification here, given that it contains a BAS factor of Goal-Drive Persistence, which is delineated from factors of Reward Interest and Impulsivity (Corr, 2016).

Another interpretation of the results is that resting-state LFA does not represent trait approach motivation per se, but rather reflects tendencies toward decreased regulatory control and impulsive tendencies (Gable, Neal, \& Threadgill, 2018; Neal \& Gable, 2017, 2019). Individuals with higher restingstate LFA in our study were more likely to select HE expenditures on lower utility offers. This pattern of choice behavior proved suboptimal, and may reflect urgency, a lack of premeditation, and/or sensation seeking - three aspects of impulsivity (Whiteside \& Lynam, 2001). Again, use of the RST-PQ may have been helpful in providing some initial data in this regard, given the survey contains an Impulsivity factor. However, the confound of self-report remains. Moreover, the existing associations between self-report impulsivity and frontal asymmetry - similar to the existing associations between self-report BAS and frontal asymmetry-are inconsistent and vulnerable to publication bias. A recent meta-analysis revealed a nonsignificant main effect size of $r=.039$ across 25 independent studies examining impulsivity and LFA (Kuper et al., 2019). Overall, making conclusive links between resting-state LFA and self-reported personality traitswhether in relation to approach motivation and/or impulsivity - appears tenuous.

What can be preliminarily concluded from our small cohort's data $(n=49)$ is that resting-state LFA is associated with — on an observable, behavioral level—a willingness to expend effort for rewards that are less certain or are of midrange magnitude. Hughes et al. (2015) reported a similar finding in their 2015 paper, in that LFA at rest (which in their study was recorded after the EEfRT) correlated to HE choice selection on low-probability trials during the task. They suggested that LFA at rest may reflect a more nuanced aspect of approach motivation than previously recognized, and our results are in agreement. We have replicated their finding regarding resting-state LFA and low-probability trials, and in 
our sample the correlation extends to also capture mediumprobability and medium-reward trials $(\beta=0.23$ and 0.16 , respectively). Interestingly, in a recent tDCS study by Ohmann, Kuper, and Wacker (2018), left frontal anodal stimulation delivered throughout the 20-min EEfRT caused an increase in HE choice percentage on low-probability trials, and the effect of tDCS on HE choice selection linearly increased alongside reward magnitude. Taken together, a heightened LFA baseline-whether as the endogenous resting-state baseline and/or as a tDCS enhanced baselineappears to increase approach motivation when large rewards are present, but so, too, are costs (effort, probability).

The results presented here also highlight a novel distinction between resting-state LFA and task-state LFA in relation to behavioral performance in the EEfRT. While resting-state LFA was statistically related to the more nuanced aspects of task performance, as discussed above, task-onset LFA correlated to overall performance in the EEfRT. We were particularly interested in examining LFA during the first $5 \mathrm{~min}$ of the EEfRT ("task-onset") for two reasons. One, we felt that these first 5 min would capture entry into task state, as compared with the 10-min resting-state, which occurred prior. Just as resting-state LFA has been studied for its capacity to predict approach-like tendencies, we wanted to test whether taskonset LFA could to some degree predict approach-like behavior in the EEfRT session. Two, we felt that these first $5 \mathrm{~min}$ would capture the participant in their most motivated state: They had just completed practice trials under experimenter guidance and demonstrated mastery of the task; they had just been informed about the $\$ 100$ gift voucher awarded to the top performer; and their hands would be in the least fatigued state of the session. In other tasks, fatigue development has been linked to changes in frontal alpha power (e.g., Tops \& Boksem, 2010; Wascher et al., 2014). Hand fatigue is suggested to occur across the 20-min EEfRT, as HE choice selection statistically decreases with trial number across the session (Hughes et al., 2015; Ohmann et al., 2018; Treadway et al., 2009).

Higher LFA during the first $5 \mathrm{~min}$ of the EEfRT did not correlate to task performance measures within those first 5 min; however, this task-onset LFA was associated with strong overall task performance in the session. Task-onset LFA in this study may therefore inform on cost-benefit decision-making strategies rather than approach motivation per se, though without further, directed experimentation this is purely speculative. LFA captured during the EEfRT may also have been influenced by the motoric finger-tapping demands of the task, as right handgrip experiments have been shown to increase LFA in some studies (Harmon-Jones, 2006), but not in other studies (Cross-Villasana, Gröpel, Doppelmayr, \& Beckmann, 2015). Thus, further investigations into any LFA aftereffects of low-effort (right-hand) and high-effort (left-hand) choices for right-handed participants in the EEfRT are warranted.
In terms of limitations, our study design may have been limited by the use of a single $\$ 100$ grocery store voucher prize for the top performer each semester, as compared with paying out dollar amounts on randomly selected EEfRT trials for each participant (Treadway et al., 2009) or offering monetary prizes for, say, the top 10 performers in a task (e.g., Tops \& Boksem, $2010)$. We initially reasoned that a $\$ 100$ voucher would be viewed as lucrative - and thus motivating - for our cohort of university students; however, this may not have been as effective if, say, at the start a participant thought their chance of being the top performer was minimal. Our study also only examined BIS/BAS and did not explore other personality traits such as social desirability, which may have influenced participants' behavior in the EEfRT and/or LFA (Pauls, Wacker, \& Crost, 2005). Participants' behavior in the EEfRT - notably their trial completion rates but also their \% selection of HE trials - could also be influenced by innate motoric abilities and dexterity, rather than motivation.

A major limitation of our study relates to sample size and statistical power. As stated in the Method section, our sample size target was set based on a previous study examining restingstate LFA and EEfRT performance (Hughes et al., 2015), which had an initial recruitment of 55 participants and reported effect sizes of .42-.43. To detect a similar effect size in our study $(r=.40,1-\beta=.80), 49$ participants would be required. Our sample was composed of 49 individuals; however, upon reviewer suggestion, we conducted post hoc power calculations and found that our study was underpowered. When examining our correlations of interest - that is, the observed correlations between resting-state and/or task-state LFA and EEfRT performance - we found that our sample size of 49 fell short in some areas. As shown in Table 6, the association between task-onset LFA and HE trial selection exceeded $80 \%$ power, as did the association between resting-state LFA and HE, medium-reward trial selection. Four of the other associations that we reported as significant achieved $75 \%-77 \%$ statistical power, while the association between task-onset LFA and total money earned in the EEfRT was the lowest with a post hoc power calculation of only $65 \%$. Unfortunately, we are no longer in a position to run additional participants or extend the study, so our data set must remain at 49 participants. Based on these identified issues surrounding statistical power, we must present the study as exploratory and the findings - and their interpretations - as preliminary.

While ours was not a large-scale study, our sample size was based on an a priori power calculation, and we believe our initial results surrounding resting-state LFA, task-state LFA, and EEfRT performance can lead to interesting future investigations. Our lack of significant correlations between BIS/BAS scores and resting-state LFA are not out of place in an increasingly mixed field: seminal studies have shown significant correlations between LFA and self-reported BAS (Coan \& Allen, 2003a; Harmon-Jones \& Allen, 1997; Sutton \& Davidson, 
1997); however, many others have failed to find such associations (for review, see Kuper et al., 2019; Wacker et al., 2010). The discrepancies could in part stem from methodological differences and self-reporting survey confounds, but also perhaps the prominence of resting-state EEG captured in the literature. Here we show that resting-state LFA and task-state LFAsrecorded from the same participant in the same recording session - inform on different performance measures in a goaloriented task, at least in our cohort of 49 participants. Our findings, in combination with other recent studies that have assessed task-state changes in LFA (e.g., Neal \& Gable, 2019; Rodrigues et al., 2018; Rollwage et al., 2017), highlight the need to make state-trait and/or state-state comparisons when assessing LFA, specifically in relation to approachavoidance observed on an emotive level or an action level (Coan \& Allen 2003b). In line with this, it is worth considering the approach-avoidance LFA literature within a broader framework of effort allocation, one that spans the emotive and action levels (Tops, Quirin, Boksem, and Koole, 2017).

Acknowledgements We thank Dr. Michael Treadway and his team for providing the EEfRT MATLAB code, and Mr. Joe Clutter for assistance with data collection. Funding to conduct this study was provided by the Department of Psychology at the University of Otago.

Open practices statement None of the experiments were preregistered, and materials availability is not applicable for this type of experiment. Data sets are available upon request to the corresponding author.

\section{References}

Allen, J. J., Coan, J. A., \& Nazarian, M. (2004). Issues and assumptions on the road from raw signals to metrics of frontal EEG asymmetry in emotion. Biological Psychology, 67(1/2), 183-218.

Amodio, D. M., Master, S. L., Yee C. M., \& Taylor, S. E. (2008). Neurocognitive components of the behavioral inhibition and activation systems: Implications for theories of self-regulation. Psychophysiology, 45(1), 11-19.

Bijttebier, P., Beck, I., Claes, L., \& Vandereycken, W. (2009). Gray’s reinforcement sensitivity theory as a framework for research on personality-psychopathology associations. Clinical Psychology Review, 29(5), 421-430.

Carver, C. S., \& White, T. L. (1994). Behavioral-inhibition, behavioral activation, and affective responses to impending reward and punishment-The Bis/Bas Scales. Journal of Personality and Social Psychology, 67(2), 319-333.

Coan, J. A., \& Allen, J. J. B. (2003a). Frontal EEG asymmetry and the behavioral activation and inhibition systems. Psychophysiology, 40(1), 106-114.

Coan, J. A., \& Allen, J. J. B. (2003b). The state and trait nature of frontal EEG asymmetry in emotion. In K. Hugdahl \& R. J. Davidson (Eds.), The asymmetrial brain (pp. 565-615). Cambridge, MA: MIT Press.

Coan, J. A., \& Allen, J. J. B. (2004). Frontal EEG asymmetry as a moderator and mediator of emotion. Biological Psychology, 67(1-2), 7-49.
Coan, J. A., Allen, J. J. B., \& Harmon-Jones, E. (2001). Voluntary facial expression and hemispheric asymmetry over the frontal cortex. Psychophysiology, 38(6), 912-925.

Coan, J. A., Allen, J. J. B., \& McKnight, P. E. (2006). A capability model of individual differences in frontal EEG asymmetry. Biological Psychology, 72(2), 198-207.

Cogswell, A., Alloy, L. B., van Dulmen, M. H. M., \& Fresco, D. M. (2006). A psychometric evaluation of behavioral inhibition and approach self-report measures. Personality and Individual Differences, 40(8), 1649-1658.

Cooper, A., Gomez, R., \& Aucote, H. (2007). The Behavioural Inhibition System and Behavioural Approach System (BIS/BAS) Scales: Measurement and structural invariance across adults and adolescents. Personality and Individual Differences, 43(2), 295-305.

Corr, P. J. (2008). The reinforcement sensitivity theory of personality. Cambridge, England: Cambridge University Press.

Corr, P. J. (2013). Approach and avoidance behaviour: Multiple systems and their interactions. Emotion Review, 5(3), 285-290.

Corr, P. J. (2016). Reinforcement sensitivity theory of personality questionnaires: Structural survey with recommendations. Personality and Individual Differences, 89, 60-64.

Corr, P. J., \& Cooper, A. J. (2016). The Reinforcement Sensitivity Theory of Personality Questionnaire (RST-PQ): Development and validation. Psychological Assessment, 28(11), 1427-1440.

Corr, P. J., \& McNaughton, N. (2012). Neuroscience and approach/ avoidance personality traits: A two stage (valuation-motivation) approach. Neuroscience and Biobehavioral Reviews, 36(10), 2339-2354.

Cross-Villasana, F., Gröpel, P., Doppelmayr, M., \& Beckmann, J. (2015). Unilateral left-hand contractions produce widespread depression of cortical activity after their execution. PLOS ONE, 10(12), e0145867.

Crost, N. W., Pauls, C. A., \& Wacker, J. (2008). Defensiveness and anxiety predict frontal EEG asymmetry only in specific situational contexts. Biological Psychology, 78(1), 43-52.

Davidson, R. J. (1984). Affect, cognition, and hemispheric specialization. In C.E. Izard, J. Kagan, \& R. B. Zajonc (Eds.), Emotions, cognition and behavior (pp. 320-365). New York, NY: Cambridge University Press.

Davidson, R. J. (1998). Affective style and affective disorders: Perspectives from affective neuroscience. Cognition and Emotion, 12(3), 307-330.

De Pascalis, V., Sommer, K., \& Scacchia, P. (2018). Resting frontal asymmetry and reward sensitivity theory motivational traits. Scientific Reports, 8, 13154.

Duckworth, A., \& Gross, J. J. (2014). Self-control and grit: Related but separable determinants of success. Current Directions in Psychological Science, 23(5), 319-325.

Duckworth, A. L., Peterson, C., Matthews, M. D., \& Kelly, D. R. (2007). Grit: Perseverance and passion for long-term goals. Journal of Personality and Social Psychology, 92(6), 1087-1101.

Franken, I. H. A. (2002). Behavioral approach system (BAS) sensitivity predicts alcohol craving. Personality and Individual Differences, 32(2), 349-355.

Gable, P. A., Neal, L. B., \& Threadgill, A. H. (2018). Regulatory behavior and frontal activity: Considering the role of the revised-BIS in relative right frontal asymmetry. Psychophysiology, 55(1), e12910.

Gelman, A. (2019). Don't calculate post-hoc power using observed estimate of effect size. Annals of Surgery, 269(1), E9-E10.

Gray, J. A. (1982). The neuropsychology of anxiety: An enquiry into the function of the septo-hippocampal system. Oxford, England: Clarendon Press.

Gray, J. A. (1993). Framework for a taxonomy of psychiatric disorder. In S. van Gozen, N. van de Poll, \& J. A. Sergeant (Eds.), Emotions: Essays on emotion theory. Mahwah, NJ: Erlbaum.

Gray, J. A., \& McNaughton, N. (2000). The neuropsychology of anxiety: An enquiry into the function of the septo-hippocampal system. Oxford, England: Oxford University Press. 
Hagemann, D., Hewig, J., Seifert, J., Naumann, E., \& Bartussek, D. (2005). The latent state-trait structure of resting EEG asymmetry: Replication and extension. Psychophysiology, 42(6), 740-752.

Harmon-Jones, E. (2006). Unilateral right-hand contractions cause contralateral alpha power suppression and approach motivational affective experience. Psychophysiology, 43(6), 598-603.

Harmon-Jones, E., \& Allen, J. J. B. (1997). Behavioral activation sensitivity and resting frontal EEG asymmetry: Covariation of putative indicators related to risk for mood disorders. Journal of Abnormal Psychology, 106(1), 159-163.

Harmon-Jones, E., Gable, P. A., \& Peterson, C. K. (2010). The role of asymmetric frontal cortical activity in emotion-related phenomena: A review and update. Biological Psychology, 84(3), 451-462.

Harmon-Jones, E., Harmon-Jones, C., \& Price, T. F. (2013). What is approach motivation? Emotion Review, 5(3), 291-295.

Hayden, E. P., Bodkins, M., Brenner, C., Shekhar, A., Numberger, J. I., O'Donnell, B. F., \& Hetrick, W. P. (2008). A multimethod investigation of the behavioral activation system in bipolar disorder. Journal of Abnormal Psychology, 117(1), 164-170.

Hewig, J., Hagemann, D., Seifert, J., Naumann, E., \& Bartussek, D. (2006). The relation of cortical activity and BIS/BAS on the trait level. Biological Psychology, 71(1), 42-53.

Hughes, D. M., Yates, M. J., Morton, E. E., \& Smillie, L. D. (2015). Asymmetric frontal cortical activity predicts effort expenditure for reward. Social Cognitive and Affective Neuroscience, 10(7), 1015-1019.

Johnson, S. L., Turner, R. J., \& Iwata, N. (2003). BIS/BAS levels and psychiatric disorder: An epidemiological study. Journal of Psychopathology and Behavioral Assessment, 25(1), 25-36.

Kuper, N., Käckenmester, W., \& Wacker, J. (2019). Resting frontal EEG asymmetry and personality traits: A meta-analysis. European Journal of Personality, 33(2), 154-175.

Laufs, H., Kleinschmidt, A., Beyerle, A., Eger, E., Salek-Haddadi, A., Preibisch, C., \& Krakow, K. (2003). EEG-correlated fMRI of human alpha activity. NeuroImage, 19(4), 1463-1476.

Lenth, R. V. (2001). Some practical guidelines for effective sample size determination. American Statistician, 55(3), 187-193.

Mognon, A., Jovicich, J., Bruzzone, L., \& Buiatti, M. (2011). ADJUST: An automatic EEG artifact detector based on the joint use of spatial and temporal features. Psychophysiology, 48(2), 229-240.

Neal, L. B., \& Gable, P. A. (2017). Regulatory control and impulsivity relate to resting frontal activity. Social Cognitive and Affective Neuroscience, 12(9), 1377-1383.

Neal, L. B., \& Gable, P. A. (2019). Shifts in frontal asymmetry underlying impulsive and controlled decision-making. Biological Psychology, 140, 28-34.

Ohmann, H. A., Kuper, N., \& Wacker, J. (2018). Left frontal anodal tDCS increases approach motivation depending on reward attributes. Neuropsychologia, 119, 417-423.

Pauls, C. A., Wacker, J., \& Crost, N. W. (2005). The two components of social desirability and their relations to resting frontal asymmetry. Journal of Individual Differences, 26, 29-42.

Pickering, A. (2008). Formal and computational models of reinforcement sensitivity theory. In P. J. Corr (Ed.), The reinforcement sensitivity theory of personality (pp. 453-481). Cambridge, England: Cambridge University Press.

Pizzagalli, D. A., Sherwood, R. J., Henriques, J. B., \& Davidson, R. J. (2005). Frontal brain asymmetry and reward responsiveness: A source-localization study. Psychological Science, 16(10), 805-813.

Rodrigues, J., Müller, M., Mühlberger, A., \& Hewig, J. (2018). Mind the movement: Frontal asymmertry stands for behavioral motivation, bilateral front activation for behavior. Psychophysiology, 55(1), e12908.

Rollwage, M., Comtesse H., \& Stemmler, G. (2017). Risky economic choices and frontal EEG asymmetry in the context of ReinforcerSensitivity-Theory-5. Cognitive, Affective, \& Behavioral Neuroscience, 17(5), 984-1001.
Schneider, M., Chau, L., Mohamadpour, M., Stephens, N., Arya, K., \& Grant, A. (2016). EEG asymmetry and BIS/BAS among healthy adolescents. Biological Psychology, 120, 142-148.

Smillie, L. D. (2008). What is reinforcement sensitivity? Neuroscience paradigms for approach-avoidance process theories of personality. European Journal of Personality, 22(5), 359-384.

Smillie, L. D., Pickering, A. D., \& Jackson, C. J. (2006). The new reinforcement sensitivity theory: Implications for psychometric measurement. Personality and Social Psychology Review, 10(4), 320-335.

Smits, D. J. M., \& Kuppens, P. (2005). The relations between anger, coping with anger, aggression, and the BIS/BAS system. Personality and Individual Differences, 39(4), 783-793.

Sutton, S. K., \& Davidson, R. J. (1997). Prefrontal brain asymmetry: A biological substrate of the behavioral approach and inhibition systems. Psychological Science, 8(3), 204-210.

Taubitz, L. E., Pedersen, W. S., \& Larson, C. L. (2015). BAS reward responsiveness: A unique predictor of positive psychological functioning. Personality and Individual Differences, 80, 107-112.

Tomarken, A. J., Davidson, R. J., Wheeler, R. E., \& Kinney, L. (1992). Psychometric properties of resting anterior EEG asymmetry: Temporal stability and internal consistency. Psychophysiology, 29(5), 576-592.

Tops, M., \& Boksem, M. A. S. (2010). Absorbed in the task: Personality measures predict engagement during task performance as tracked by error negativity and asymmetrical frontal activity. Cognitive, Affective, \& Behavioral Neuroscience, 10, 441-453.

Tops, M., Quirin, M., Boksem, M. A. S., \& Koole, S. L. (2017). Largescale neural networks and the lateralization of motivation and emotion. International Journal of Psychophysiology, 119, 41-49.

Torrubia, R., Avila, C., Molto, J., \& Caseras, X. (2001). The Sensitivity to Punishment and Sensitivity to Reward Questionnaire (SPSRQ) as a measure of Gray's anxiety and impulsivity dimensions. Personality and Individual Differences, 31(6), 837-862.

Treadway, M. T., Buckholtz, J. W., Schwartzman, A. N., Lambert, W. E., \& Zald, D. H. (2009). Worth the 'EEfRT'? The Effort Expenditure for Rewards Task as an objective measure of motivation and anhedonia. Plos One, 4(8), e6598.

Vuga, M., Fox, N. A., Cohn, J. F., George, C. J., Levenstein, R. M., \& Kovacs, M. (2006). Long-term stability of frontal electroencephalographic asymmetry in adults with a history of depression and controls. International Journal of Psychophysiology, 59(2), 107-115.

Vuga, M., Fox, N. A., Cohn, J. F., Kovacs, M., \& George C. J. (2008). Long-term stability of electroencephalographic asymmetry and power in 3 to 9 year-old children. International Journal of Psychophysiology, 67(1), 70-77.

Wacker, J., Chayanon, M. L., \& Stemmler, G. (2010). Resting EEG signatures of agentic extraversion: New results and meta-analytic integration. Journal of Research in Personality, 44(2), 167-179.

Wacker, J., Mueller, E. M., Pizzagalli, D. A., Hennig, J., \& Stemmler, G. (2013). Dopamine-D2-receptor blockade reverses the association between trait approach motivation and frontal asymmetry in an approach-motivation context. Psychological Science, 24(4), 489-497.

Wascher, E., Rasch, B., Sänger, J., Hoffmann, S., Schneider, D., Rinkenauer, G., Heuer, H., \& Gutberlet, I. (2014). Frontal theta activity reflects distinct aspects of mental fatigue. Biological Psychology, 96, 57-65.

Whiteside, S. P., \& Lynam, D. R. (2001). The five factor model and impulsivity: Using a structural model of personality to understand impulsivity. Personality and Individual Differences, 30(4), 669-689.

Publisher's note Springer Nature remains neutral with regard to jurisdictional claims in published maps and institutional affiliations. 